\title{
Development of 6LoWPAN Border Router for Secure Communication
}

\author{
Mr. Tejas Mehare', Prof. Mrs. Snehal Bhosale ${ }^{2}$ \\ Student (ME), Electronics and Telecommunication, R.M.D. Sinhgad School of Engineering, Pune, India ${ }^{1}$ \\ Professor, Electronics and Telecommunication, R.M.D. Sinhgad School of Engineering, Pune, India ${ }^{2}$
}

\begin{abstract}
Accessing end devices (nodes) in the Internet of things (IoT) with a unique IP address is possible with the evolution IPv6 and 6LoWPAN. which is an open stack developed by IETF to provides communication between LoWPAN devices and the internet. 6LoWPAN Border Router is the gateway between nodes and internet which connects 6LoWPAN devices to the Internet and also responsible for controlling traffic between IPv6 and IEEE 802.15.4 interfaces. 6LoWPAN Networks are implemented by several ways, but some are open standards which can be executed on development boards like Raspberry Pi, Beagle bones etc. The design and implementation of 6LoWPAN border router with an embedded Web server and implement the bridge between 6LoWPAN devices to the internet (IPv4 as well as IPv6). The border router is built around a 32-bit ARM Cortex M3 microcontroller and runs the network-enabled operating system Contiki in version 2.7.The network layer uses IPv6 and Layer-3 forwarding between these different link-layer technologies.
\end{abstract}

Keywords: Internet of Things, 6LoWPAN Border Router, IPv6, IEEE 802.15.4, Contiki-OS.

\section{INTRODUCTION}

Internet of Things (IoT) applications are implemented using a wide range of proprietary technologies which are difficult to integrate with larger networks and Internetbased services, whereas 6LoWPAN approach is IP based one, these devices can be connected easily to other IP networks, which doesn't require any translation gateways or proxies, and which can use the existing network infrastructures.

Many researchers and market participants expect that the next revolution of the Internet comes from the interconnection of many, so-called smart objects. These are unconspicuous electronic devices, equipped with sensors or actuators, a microcontroller, and a communication device that will form the backbone of the Internet of Things [1], [2].

These smart objects have to be integrated into real network infrastructures mainly through a wireless link layer. A good candidate is the Low-Power WPAN IEEE 802.15.4 [3], which has been designed to provide low-bit rate network connectivity efficiently and at minimal cost. It can be used with different network stacks such as Zigbee or IPv6 [4]. The Internet of Things will have to use IPv6, which has a collection of 2128 addresses and offers more than enough room to grow the Internet into the physical world. In order to run IPv6 on IEEE 802.15.4 links, with a frame size of 127 octets, an adaptation layer is unavoidable that provides link-specific fragmentation and reassembly, as well as header compression. This service is provided by 6LoWPAN [5].

6LoWPANs are usually stub networks that work with resource constrained devices (memory, processing power, and energy). It is, therefore, important to reduce packet overhead, and bandwidth utilization, as well as processing requirements. RFC 6568 suggest the concept of a LoWPAN Border Router (LBR) [6] that is responsible for network coordination, address configuration, and network interconnection.

In past five decades, we have come across various technological waves. At first, it was the advent of Computers in our daily lives. People became astonished by the GUIs offered by Windows 98, and then Windows XP and beyond. The Upward trend of beautifying didn't stop. Then came the Internet, although it was there for soldiers, it became famous very recently. Things like Social-media, chatting sites, all such fancy toys, made it popular. Now we are living in a time where a third wave is in its infancy. Something which was glamorized in Science-Fictions. That third wave is called as Internet of things (IoT). We can have any possible thing on Internet with this technology. And they will take part in giving the judgment. It won't even require Human involvement. And a part of this network contains Low-Power and constrained devices, like small miniaturized computers, with 8 bit to 16-bit processors. Combine this type of Network (Internet of things) with the Network which we are already familiar with will bring some challenges. Challenge is a mother of innovations and inventionsBorder Router became the new binding force.

In order to use IoT network, installing a Border Router (BR) is a must. Border Router is a gateway to 802.15.4 (WPAN) tools and Ethernet or Wi-Fi on another side. One such Border router is a 6LoWPAN Border router (6LBR). 


\section{RELATED WORK}

Currently, there does not exist any freely available and aplicable implementation of a 6LoWPAN stack for desktop operating systems such as Linux or Windows. The Linux ZigBee project aims to provide a complete execution of the IEEE 802.15.4 and 6LoWPAN protocol. However, progress has been relatively slow and only recently has it become possible to exchange basic IPv6 messages between the Linux stack and Contiki. The current prototypical RPL implementation for Linux Simple RPL has only limited functionality and is not yet able to communicate successfully with other 6LoWPAN implementations due to kernel issues. Hence, all notable RPL border router application still use the Contiki 6LoWPAN stack in different configurations:

A possible solution for the formation of a 6LoWPAN border router requires RZRaven USB stick4 from Atmel, which runs a complete Contiki installation. The stick can be installed as a network combined on a computer running a desktop operating system such as Linux or Windows. The device then acts as a link between the WPAN and the global IPv6 Internet [7]. Limitations are the low number of possible RPL routes that this solution supports (due to the low memory size of $8 \mathrm{kByte}$ on the Atmega AT9OUSB1287) and the cumbersome network configuration that requires the addition of explicit MACaddresses into the neighbor table for certain operating systems.

Beerli and Fischer [8] proposed a solution Contiki platform has native Ethernet interface support and a microcontroller with more memory and processing power than the Atmel MCU. However, this design is not openly available and uses Contiki to bridge packets between the Ethernet and the IEEE 802.15.4 network, instead of routing them at Layer 3. A third implementation access for a 6LoWPAN border router is chosen by the 6LBR project [9]. They use the Contiki OS compiled as a native process on Linux to handle the border router task. This approach gives the most opportunity from resource constraints; however it also requires a much more powerful hardware platform to run. The code could also be run on a microcontroller, but would require a porting effort, especially since their works very much focus point on the provisioning of multiple border routers to provide redundant points of attachment for the 6LoWPAN.

We choose to follow the path taken by Beerli and Fisher and advanced our own hardware platform. However, we also want to make the necessary modifications to Contiki to support packet forwarding and routing between interfaces with particular hardware address sizes. We also intent to open-source our implementation, that can be used as stand-alone RPL border router by other projects.

The network layout and the packet rewriting process that Devices to the left of the border router are normal IPv6 network nodes that connect via Ethernet with the border router. Devices to the right of the border router run a RPLimplemented 6LoWPAN stack. We have successfully tested our prototype with several sensor nodes running Contiki in a multi-hop configuration.

\section{BACKGROUND}

In this section, we give an overview of 6LoWPAN Border Router, 6LBR Modes and routing in 6LoWPAN.

\section{A. Overview of 6LoWPAN Border Router}

6LBR is placed in between Access point and IPv6 only nodes, packets from internet to nodes are adopted via 6LoWPAN Border Router and which also responsible for converting 6LoWPAN to IPv6 and vice-versa.

6LBR architecture includes IPv6[rfc 2460] for circulating unique IP's to the nodes, 6LoWPAN stack for implementing Radio to establish communication between node and cloud,NAT64 $\mathrm{v}^{9}$ for internet interoperability with node. Architecture of 6LBR is shown in Figure 1.

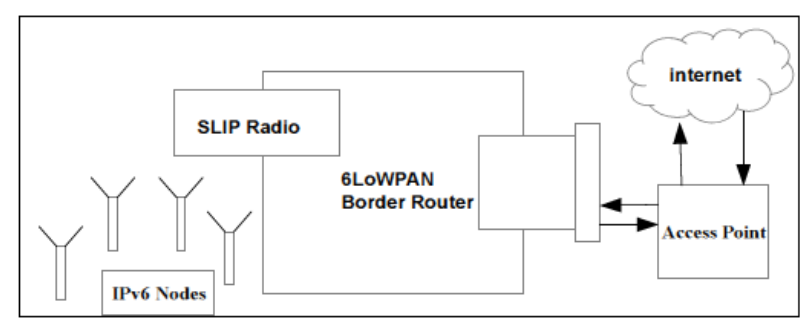

Fig 1. General architecture for 6LBR

\section{B. 6LoWPAN Border Router Modes}

6LBR runs in three categories of modes: bridge, router and transparent bridge. These three categories are declined into the following modes :

\section{Smart Bridge Mode:}

In this mode, the 6LBR acts as a Smart bridge, allowing interconnecting a standard IPv6 based network with a RPL based WSN mesh. The Smart Bridge is acting as a NDP proxy on the Ethernet side and is using NDP parameters to configure the WSN mesh. Source and destination MAC addresses are adapted and addresses present in ICMPv6 packets are also translated. The Smart Bridge mode provides:

- Flawlessly incorporate a WSN mesh into an actual NDP based IPv6 network, the Smart Bridge will use the NDP provided configuration to set up the WSN mesh accordingly.

- Aggregate several WSN meshes with their own DODAG into one essential IPv6 subnet. When two WSN coincide based on node mobility, one node can convert from one WSN to the other as observed from the virtual IPv6 subnet, this swap will be almost undetectable thanks to the NDP proxy.

\section{Router mode:}

In this mode, the 6LBR acts as a full fledged IPv6 Router, interconnecting two IPv6 subnets. The WSN subnet is handled by the RPL protocol and the Ethernet subnet is managed by IPv6 NDP. In this mode, the 6LBR provides a 
virtual second interface to Contiki thanks to the packet technologies such as Ethernet or WiFi, that provide a filter module. This mode acts more like a Gateway single newscast domain. Both approaches have pros and between Ethernet and 6Lowpan RPL. Open packets source corns. The most prominent drawback of the mesh-under address from RPL side will be updated as approach is the high overhead associated with the planning eth_ip_local_addr. of a multicast service that backing the IPv6 neighbor discovery protocol and the hidden topology of the

The Router moder allows you to :

- Isolate WSN mesh into its own subnet, therefore clearly determine the WSN nodes.

- Node mobility across different WSN subnets is supported thanks to the prefix switching capacity. In that case the nodes will get a new address, based on the adjunct of the new WSN.

\section{Transparent Bridge Modes:}

In these modes, the 6LBR acts as a standalone bridge, providing basic switching capabilities. 802.15.4 Packets addressing an Ethernet interface are transfer to the WSN segment. Conversely, all incoming packets addressing an Ethernet interface or incoming multicast packets on the 802.15.4 interface are delivered to the Ethernet segment. The 6LBR has its individual address and act as a host. Source and destination MAC addresses are adapted and addresses present in ICMPv6 packets are also reworded. The transparent Bridge Mode allows you to:

- Total sub-WSN meshes into DODAG, handled by an external RPL Root node (When using RPL-Relay)

- Bridge a one-hop mesh with an IPv6 network using NDP (When using Full Transparent Bridge)

- Bridge a statically routed mesh with an IPv6 network (When using Full Transparent Bridge).

\section{ROUTING IN 6LoWPANs}

IEEE 802.15.4 network devices use low power radios, that imply a typical signal range in tens of meters, and even less in riotous and obstructed environments. Therefore the standards perceive the support for mesh plot, where two devices do not require direct reach ability in order to communicate. However, neither IEEE 802.15.4 nor RFC 4944 define mechanisms for the operation and management of such mesh networks.

Given the key design of network nodes in 6LoWPANs, several challenges exist: devices may be battery powered and enforced on microcontrollers with just a few kByte of RAM. RFC 6606 cites the following test for mesh networking in 6LoWPANs [10]:

- low overhead on data packets

- low routing overhead

- minimal memory and calculation requirements

- support for sleeping nodes (saving battery)

There exist two principal approaches for packet routing in 6LoWPANs: route-over and mesh under. The route-over approach routes packets on the IP level which has the implication that intermediate nodes in the network have to make forwarding result. The mesh-under approach treats the 6LoWPAN mesh as a single IP hop, similar to network network, which prevents the IP and application layer to optimize performance. Because of these conditions, almost all current implementations (including ours) implement a route-over solution.

The route-over approach also provides some challenges. The 6LoWPAN is a single IPv6 subnet and regular prefixbased forwarding does not work. Intermediate routers might experience temporary link loss due to changing channel conditions, node mobility or sleeping devices. Packet delivering in a 6LoWPAN is usually done over a single wireless interface. Devices have very little memory and any routing protocol will have to minimize state.

The IETF advanced the Routing Protocol for Low-Power and Lossy Networks (RPL) for networks with extremely constrained resources. It works under the assumption that a few administratively chosen devices form the source of a directed acyclic graph (DAG). Routes exist as up and down routes and also transitional nodes in the 6LoWPAN can act as routers to provide multi-hop forwarding beyond the radio coverage of the DAG root.

Depending on the memory situation of the devices, RPL can be run in storing or non-storing mode. Transitional nodes in non-storing mode do not keep downward routes. They only have enough memory to keep state about their connected parents and propagate information about connected children systematically in the form of a destination advertisement object (DAO) to the root node. The root node collects all the individual parent information and calculates reachability information for the whole 6LoWPAN. When a packet needs to be sending to a node within the 6LoWPAN, the root inserts appropriate source-routes into the packet so that intermediate nodes can make adequate forwarding decisions without having to manage a full routing table.

In storing mode the median nodes keep downward routing data in their routing table and transmit only the targeting addresses and prefixes for which a node has routes. Upward routes are handled as default routes chosen during the parent selection process. The Contiki OS [11] is an open source operating system that helps a large variety of devices and has a tiny memory footprint, which allows it to run on 8bit microcontrollers with 8 kByte RAM and upwards of 30 kByte ROM. The Contiki OS is written in $\mathrm{C}$, so it becomes relatively easy to port it to alternative hardware platforms and reuse existing application code. Since version 2.7 the Contiki OS supports the RPL routing protocol, which is operating in storing mode.

\section{PROPOSED ALGORITHM}

For the designing of 6LBR we use Contiki OS 2.7. This provide introduction of Border router in Contiki OS. Border router or edge router is connected to internet (see 
fig. 2) when the other sensor nodes are reporting the data via the border router. Implementation of this router is giving by all IOT operating systems.

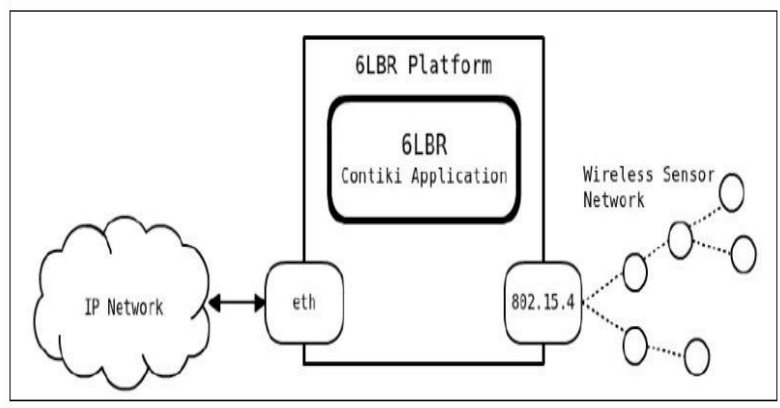

fig 2. 6LBR Model

Using this border router, how to transmit data to the internet, how neighbors are understood and how the routes are computed. We will be using the following files

1. Border-router.c

2. udp-server.c

3. slip-bridge.c

4. httpd-simple.c

udp-server nodes will form a DAG with the border router set as the root. The border router will receive the prefix through a SLIP (Serial Line Interface Protocol) connection and it will be communicated to the remaining of the nodes in the RPL network.

In the border-router.c file, the portion of code the node configured as the border router waits for the prefix to be set. Once it receives, the border router is set as the root of the DAG after which it sets the prefix of the rest of the nodes in the network.

By default the border router host a simple web page. However, this can be disabled by defining WEBSERVER. This webpage is displayed when the IPv6 address of the border router is entered in the browser.

\section{CONCLUSION}

We developed a simulation that extends the current routing functionality found in the Contiki operating system and implemented it in a working model that can be directly attached to an Ethernet segment. Our border router might be used to easily build meshed WPANs that can incorporate a large number of smart objects within a geographic area and make them universally reachable. We are currently investigating the long term stability and actual resource requirements of our solution. We expect this solution to proportion much better than current solutions, based on the Atmel Raven USB stick, mainly because the ARM Cortex M3 provides 8-times more RAM than the Atmel MCU. We also expect our solution to be much more energetic than a Linux or Windowsbased installation.

The IETF Home Network working group is currently developing a set of standards that aid the composition of routed networks in residential homes. We monitor these standards and investigate if they could be executed on the 6LoWPAN border router.

Finally, we also investigate security problems. It might be very easy for an attacker to overpower the network, because 6LoWPANs have very limited resources. We currently evaluate security requirements and inspecting how message filtering and rate-limiting on the gateway router can protect the smart objects and the surrounding network from resource exhaustion attacks.

\section{REFERENCES}

[1] Viktor Eichmann, Thomas Schaller," Development of a Contiki border router for the interconnection of 6LoWPAN and Ethernet" by Thomas Scheffle on 09 December 2014.

[2] J.-P. Vasseur and A. Dunkels, Interconnecting Smart Objects with IP: The Next Internet. Morgan Kaufmann, 2010.

[3] J. Gutierrez, M. Naeve, E. Callaway, M. Bourgeois, V. Mitter, and B. Heile, "IEEE 802.15.4: A developing standard for low-power low-cost wireless personal area networks," IEEE Network Magazine, vol. 15, no. 5, pp. 12-19, September/October 2001.

[4] K. Pister, R. Stria, J. Vasseur, and R. Alexander, "RPL: IPv6 Routing Protocol for Low-Power and Lossy Networks," Internet Engineering Task Force, RFC 6550, Mar. 2012.

[5] N. Kushalnagar, G. Montenegro, and C. Schumacher, "IPv6 over Low-Power Wireless Personal Area Networks (6LoWPANs): Overview, Assumptions, Problem Statement, and Goals," Internet Engineering Task Force, RFC 4919, Aug. 2007.

[6] E. Kim, D. Kaspar, and J. Vasseur, "Design and Application Spaces for IPv6 over Low-Power Wireless Personal Area Networks (6LoWPANs)," Internet Engineering Task Force, RFC 6568, Apr. 2012.

[7] A. Dunkels, "Jackdaw RNDIS RPL border router," Tech. Rep., 2012.

[8] U. Beerli and S. Fischer, "Ethernet to WPAN gateway with IPv6 support," Zitricher Fachhochschule, Tech. Rep., 2009.

[9] L. Deru, S. Dawans, M. Ocaila, B. Quoitin, and 0. Bonaventure, "Redundant Border Routers for Mission-Critical 6LoWPAN Networks," in Pmceedings of the Fifth Workshop on Real-World Wireless Sensor Networks, 2013.

[10] E. Kim, D. Kaspar, C. Gomez, and C. Bormann, "Problem Statement and Requirements for IPv6 over Low-Power Wireless Personal Area Network (6LoWPAN) Routing," Internet Engineering Task Force, RFC 6606, May 2012.

[11] Dunkels, B. Gronvall, and T. Voigt, "Contild - A Lightweight and Flexible Operating System for Tiny Networked Sensors," in Pmceedings of the 29th Annual IEEE International Conference on Local Computer Networks, ser. LCN '04. Washington, DC, USA: IEEE Computer Society, 2004, pp. 455-462.

\section{BIOGRAPHIES}

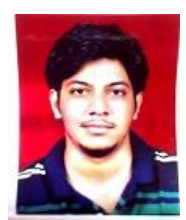

Mr. Tejas Mehare, currently studying ME in VLSI and Embedded system at RMD Sinhgad School of Engg. Pune. The author has his personal field of interest in domain of Internet of Things.

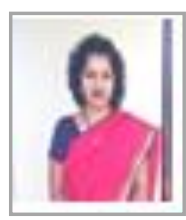

Prof. Mrs. Snehal Bhosale, currently heading Department of E\&TC at RMD Sinhgad School of Engg. Pune. Her main research interests are computer networks, network security and wireless sensor networks. She is currently doing her research on security in IoT in Pune University. 